\title{
Displasia congénita de la válvula tricúspide (anomalía de ebstein). A propósito de un caso en varón de 40 años.
}

Congenital dysplasia of the tricuspid valve (Ebstein's anomaly). In connection with a case in man of 40 years.

Steffania Renata Montesdeoca Santana. ${ }^{1}$, Gema Gabriela Vera Loor. ${ }^{2}$, Fernando Antonio Aguirre Palacios. ${ }^{3}$, Elsie Valdiviezo Valenzuela. ${ }^{4}$

Recibido: 05-06-2017 / Revisado: 12-08-2017 Aceptado: 09-09-2018/ Publicado: 01-10-2018

Abstract.

DOI: https://doi.org/10.33262/cienciadigital.v2i4.221

The Ebstein anomaly is a rare congenital heart disease, of isolated presentation in adults, with an incidence of approximately $0.03 \%$ of all congenital heart diseases, which can occur isolated or associated, characterized by the anomalous implantation of the tricuspid valve, producing insufficiency; the form and age of presentation depends on the degree of valvular dysfunction and size of the ventricle. We present the case of a 40-year-old man who reports having been diagnosed 10 years ago of suspected congenital heart disease whose name he does not know, refusing treatments and controls at that time, which now enters emergency functional level III / IV of the NYHA, the physical examination suggests tricuspid insufficiency, and cardiomegaly so it is admitted for treatment and study in which the presence of congenital severe tricuspid valve dysplasia or isolated Ebstein anomaly is confirmed by transthoracic and transesophageal echocardiography.

\footnotetext{
1 Universidad Católica de Santiagode Guayaquil, Médico General, Guayaquil, Ecuador,steff.montesdeoca@gmail.com

2 Universidad de Guayaquil, Médico General, Guayaquil, Ecuador, g.vera.85@outlook.com

3 Médica especialista en Cardiología, Ecocardiografía, Guayaquil Ecuador, docheart00@ hotmail.com

$4 \quad$ Médica especialista en Cardiología, Ecocardiografía, Guayaquil Ecuador, elsyvaldi@ hotmail.com
} 
Keywords: Ebstein's anomaly, congenital heart disease, adult, tricuspid valve dysplasia.

\section{Resumen.}

La anomalía de Ebstein es una cardiopatía congénita rara, de presentación aislada en adultos, con una incidencia de aproximadamente $0.03 \%$ de todas las cardiopatías congénitas, que puede presentarse aislada o asociada, caracterizada por la implantación anómala de la válvula tricúspide produciendo insuficiencia de la misma; la forma y edad de presentación dependen del grado de disfunción valvular y tamaño del ventrículo. Se presenta a continuación el caso de un varón de 40 años quien refiere haber sido diagnosticado hace 10 años de una presunta cardiopatía congénita cuyo nombre desconoce, negándose a tratamientos y controles en ese momento, que ahora ingresa por emergencia en grado funcional III/IV de la NYHA, la exploración física sugiere insuficiencia tricuspídea, y cardiomegalia por lo que es ingresado para tratamiento y estudio en el que se confirma por ecocardiografía transtorácica y transesofágica la presencia de displasia congénita de válvula tricúspide severa o anomalìa de Ebstein aislada.

Palabras Claves: Anomalía de Ebstein, Cardiopatía congénita,Adulto, Displasia valvular tricuspídea.

\section{Introducción.}

La anomalía de Ebstein es una cardiopatía congénita rara, de presentación aislada en adultos, descrita por primera vez por dicho autor en 1866 como el desplazamiento apical de la inserción de la válvula tricúspide, más ensanchamiento del anillo fibroso, viéndose afectadas las valvas septal y posterior que a su vez se encuentran deformadas y se adhieren de forma variable al tabique interventricular por debajo de la unión auriculoventricular, resultando en una gran dilatación de la aurícula derecha, y un ventrículo derecho muy pequeño e insuficiente, junto a la atrialización de su parte superior.(1,2,5,11). El grado de disfunción valvular y tamaño del ventrículo determinan el debut de los síntomas y por tanto su edad de diagnóstico. Importante recalcar que la anomalía de Ebstein puede presentarse aislada o asociada a otras malformaciones cardíacas. Actualmente su incidencia es aproximadamente de $0.03 \%$ de todas las cardiopatías congénitas, es decir 1/ 20.000 recién nacidos vivos.(11) Debido al inusual hallazgo no solo de la patología como tal sino también de la anomalía en paciente adultos, presentamos caso clínico de un varón de 40 años quien refiere haber sido 
diagnosticado hace 10 años de presunta cardiopatía congénita cuyo nombre desconoce, negándose a tratamientos y controles en ese momento, que ahora acude por emergencia en grado funcional III/IV de la NYHA, y es ingresado para tratamiento y durante su ingreso mediante ecocardiografía transtorácica y transesofágica se confirma la presencia de implantación anómala de la válvula tricúspide, dando el diagnóstico de Anomalìa de Ebstein aislada.

\section{Caso Clínico.}

Se presenta a continuación paciente masculino de 40 años de edad, de nacionalidad ecuatoriana, habitante de la zona rural, dedicado a la agricultura, quien refiere como único antecedente de importancia haber sido diagnosticado hace 20 años de una presunta cardiopatía congénita cuyo nombre desconoce, luego de ingreso hospitalario por cuadro clínico de taquicardia y disnea súbita, negándose a tratamientos y controles en ese momento. Refiere acudir en múltiples ocasiones a emergencia por cuadro clínico similar durante los dos últimos años, y solo solicitar oxigenoterapia, y alta voluntaria. Actualmente ingresa por emergencia pálido, frío, sudoroso, disneico, taquicárdico, categoría funcional III/IV de la NYHA, a la exploración física paciente de biotipo morfológico normolíneo, afebril, presión arterial 180/90, taquipneico 28 respiraciones por minuto, con alteración de la saturación $79 \%$ que mejora mínimamente luego de la administración de oxígeno $81 \%$, presencia de acropaquias y leve cianosis distal, taquicárdico 125 latidos por minuto, y al realizar el electrocardiograma llama la atención taquicardia supraventricular con QRS aberrantes, (fig. 1) por lo que se decide administrar una dosis de digoxina intravenosa, disminuyendo la frecuencia cardiaca eventualmente, para luego ser ingresado a unidad de cuidados intensivos coronarios para monitorización continua, y continuar medicación de mantenimiento, infusión de amiodarona dosis de impregnación y posterior dosis de mantenimiento, atenolol 50mg BID, furosemida 20mg BID IV, espironolactona 25mg QD, ivabradina 7.5mg BID, resultados de exámenes de laboratorio no reportan alteración electrolítica, pero si elevación mayor a 2000 del marcador proBNP que nos hablan de insuficiencia cardiaca, a la espera de PCR de Tripanozoma Cruzi que luego se reporta como No Reactivo, realizado como protocol por provenir de una zona endémica, en la radiografía PA de tórax se observa gran 
cardiomegalia con Índice Cardiotorácico (ICT) mayor 0.65 compatible con cardiomegalia grado IV/IV acompañado de pobre definición de vasos pulmonares(fig. 2)

Continuando con la exploración física a la auscultación pulmonar, el murmullo vesicular se encontraba moderadamente disminuido y la palpación del precordio evidenciaba signo de choque de punta, además de frémito sistólico en foco tricuspídeo; los tonos cardíacos se auscultaban arrítmicos, y un soplo pansistólico de alta frecuencia sugestivo de insuficiencia tricuspídea (IT), en el nuevo electrocardiograma de control llama la atención ritmo sinusal, ondas p pulmonares compatible con aumento de tamaño auricular, frecuencia cardiaca de 60/1pm, pero reportada como 120 por reporte de ondas P como segment QRS, ademñas segment QRS ancho, y bloqueo de rama derecha. Se procede a realizar ecocardiograma transtorácico $2 \mathrm{~d}$ y doppler y ecocardiografía transesofágico que pone de manifiesto levocardia, corazón tetracameral, conexión atrioventricular y ventriculoarterial concordantes, grandes arterias normosituadas con flujos de salida ventricular normal, y drenaje de cavas y venas pulmonares normal, la membrana de la fosa oval abombaba en sístole hacia la aurícula izquierda y no existía cortocircuito en esta localización, visualizándose integridad del septum interventricular en sus segmentos peri-membranoso, trabecular y muscular, ventrículo izquierdo con diámetros severamente disminuidos y función sistólica 50\%, además se reporta baja implantación de la válvula tricúspide con atrialización de ventrículo derecho, ventrículo derecho funcional en un 35\%, hipoplásico, e insuficiencia tricuspídea severa, valva anterior especialmente displásica.(fig 3-4)

Confirmando de ésta manera el diagnóstico de Anomalía de Ebstein severa aislada. En resúmen nos encontramos frente a una displasia severa de la válvula tricuspídea no acompañada de ninguna otra malformación cardíaca, en un paciente adulto de 40 años que debuta hace 20 años con síntomas leves más controlables y con mejor pronóstico que el debut actual, presentando al momento alto riesgo de muerte súbita, y no por diagnóstico tardío o mal manejo sino por decisión del paciente. 
Gráfico No.1. Electrocardiograma. Frecuencia cardiaca: 125lpm, no se logra observar p, presencia Taquicardia Supraventricular con QRS anchos aberrantes.

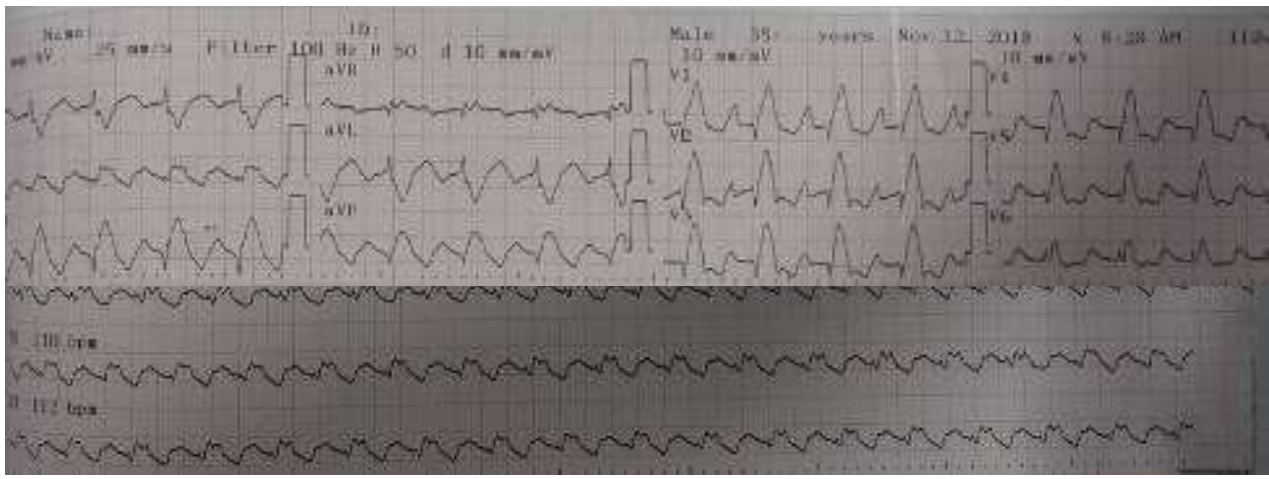

Elaborado por: Grupo de investigación.

Gráfico No.2. Radiografía de tórax. Cardiomegalia severa con ICT $(a+b / c)$ mayor 0.65 . Acompañada de disminución de trama vascular pulmonar.

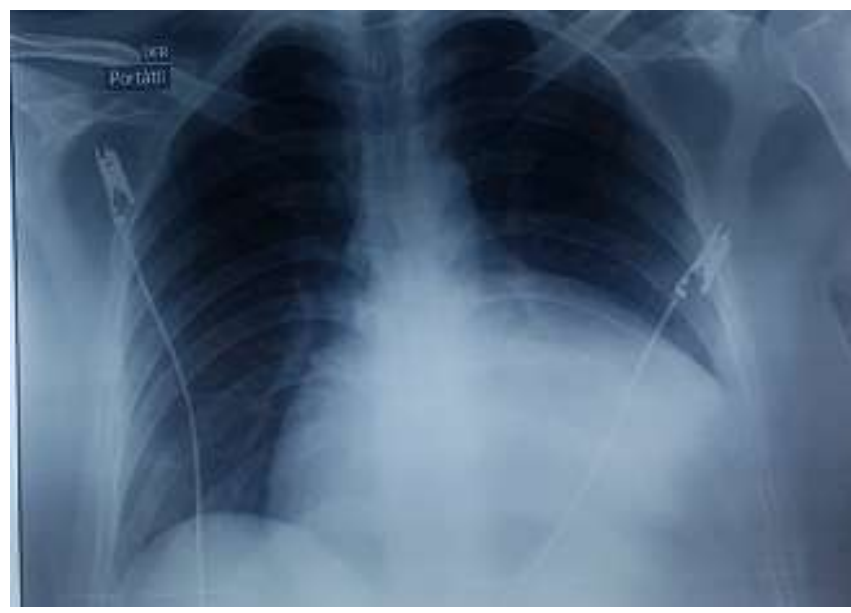

Elaborado por: Grupo de investigación. 
Gráfico No.3. Ecocardiografía transtorácica 2D. Aurícula derecha aumentada de tamaño, parte superior de ventrículo derecho atrializado, implantación apical de valvas de válvula tricúspide, con disminución del diámetro del mismo. Sin reporte de comunicación interauricular o

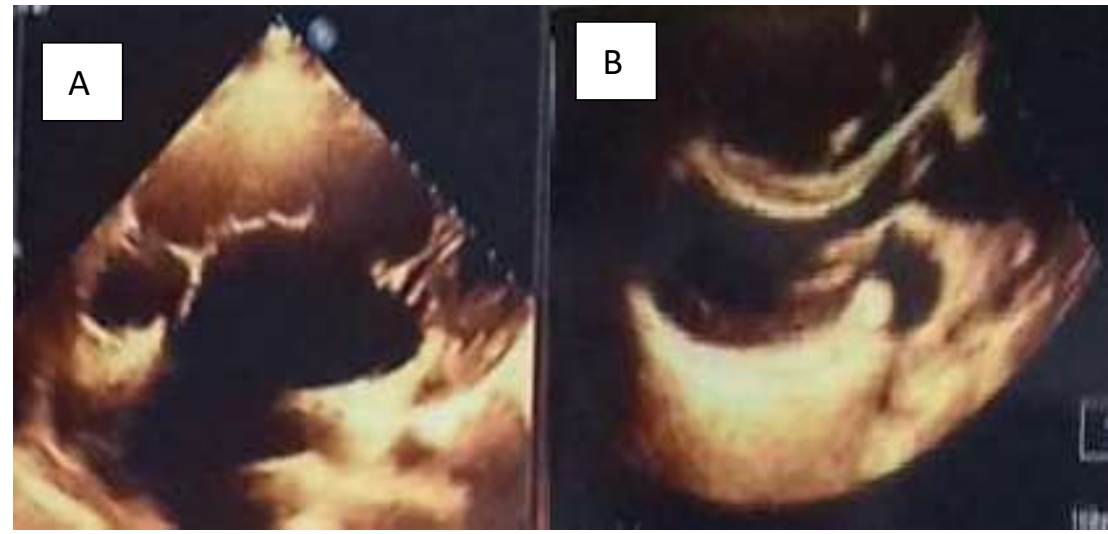

Elaborado por: Grupo de investigación.

Gráfico No.3. Ecocardiografía Transesofágico: Anomalía de Ebstein severa co Ventrículo derecho funcional del 35\%, ventrículo izquierdo hipoplásico con función sistólica levemente disminuida, insuficiencia tricuspídea severa en válvulas especialmente la anterior displásica, pobre colapso de la vena cava inferior, no se visualiza nipor técnica 2D ni 3D defecto septal interatrial.

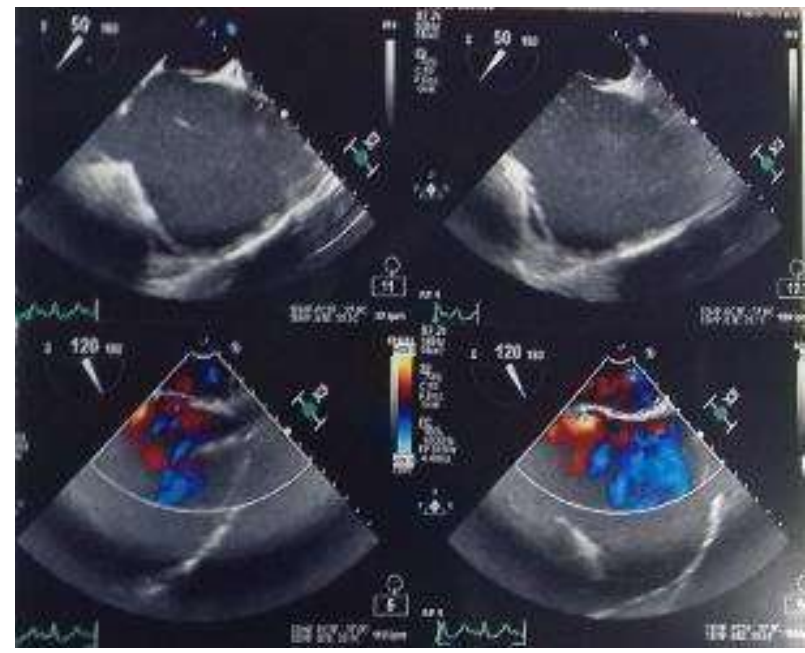

Elaborado por: Grupo de investigación. 


\section{Revisión de la enfermedad.}

Desde el punto de vista embriológico esta anomalía se relaciona a la anormalidad en el proceso de desarrollo de los cojines inferior y lateral derecho del canal aurículo ventricular y de la cresta conal dextro-dorsal o cualquier daño en el proceso de diverticulización de las paredes ventriculares. La presentación de la anomalía de Ebstein es variable. (6) Durante la vida fetal puede manifestarse como taquiarritmia, hidrops fetal secundario al fallo tricuspídeo severo, finalizando en muerte fetal en la mayoría de los casos. Mientras que en el lactante o niño mayor, la presentación más habitual es la insuficiencia cardíaca presentado menor mortalidad que en el neonato, otras formas posibles de presentación son las palpitaciones secundarias a taquiarritmias por vías accesorias en el contexto de un Síndrome de WolffParkinson White (WPW) o presencia de soplo sistólico a la exploración física. (11) En el adolescente y adulto la presentación puede incluir disnea y fatiga durante el ejercicio, provocada por su insuficiencia tricuspídea severa y por tanto disfunción ventricular derecha, además puede presentarse en 20 a $42 \%$ arritmias, siendo la forma más frecuente de debut como palpitaciones secundarias a la presencia de preexcitación, fibrilación auricular o flutter,y ocasionalmente detectada como gran cardiomegalia por radiografía de tórax en un paciente asintomático. $(\mathbf{1 , 2 , 7 )}$

El diagnóstico es posible mediante ecocardiografía ya sea, rutinaria a las 18-20 SG o parte del método diagnóstico sugerido por la clínica en cualquier grupo etario. Así mismo en el período neonatal puede debutar como cianosis grave, acidosis metabólica, insuficiencia tricuspídea grave, y cardiomegalia, por lo que el tratamiento estará orientado a la administración de prostaglandinas E1, con la finalidad de mantener el ductus arterioso abierto hasta lograr disminuir la presión pulmonar, por disminución de la misma en el ventrículo derecho, y consecuentemente mejorar la cianosis. La mortalidad en estos pacientes es muy alta y requiere intervención quirúrgica durante los primeros 10 a 15 días. $(\mathbf{6 , 1 1})$

En adultos el electrocardiograma suele presentar bloqueo de rama derecha, segmento QRS de bajo voltaje, bloqueo de primer grado, y onda $\mathrm{P}$ picudas. Además taquicardia supraventricular (TSV), flutter o fibrilación auricular, 5-10\% síndrome de WPW generalmente por vía anterolateral derecha, y sobretodo taquicardia ventricular.(3) La 
ecocardiografía ha desplazado a los estudios invasivos angiodinámicos, estando éstos indicados en casos concretos en los que se plantean dudas que no han podido ser resueltas por medio del ecocardiografía. Posicionándose como método diagnóstico de elección junto a la clínica. Regularmente encontramos levocardia, grandes vasos normalmente relacionados, y concordancia tanto atrioventricular como ventrículoarterial, se procede a la evaluación del grado de desplazamiento valvar de la válvula tricúspide, siendo la valva anterior regularmente normal, excepto en casos de displasia del total de la válvula, mientras que la septal adosada a la pared ventricular, generalmente se observa una aurícula muy dilatada producto también de un ventrículo atrializado, y la valva tricúspide elongada, largamente adosada al tabique interventricular. En la forma severa que es más frecuente, la valva posterior se encuentra aplicada a la pared ventricular y existe una valva septal rudimentaria, ambas pueden presentar diferentes grados de displasia, además de las cuerdas tendinosas y músculos papilares. Se utiliza el doppler-color para evaluación de la severidad de la IT y presencia de defecto septal interauricular. $(\mathbf{1 , 2 , 4 , 8 )}$ El tratamiento médico de la anomalía de Ebstein pauta la importancia de disminuir la precarga en el neonato muy sintomático, mientras que en el paciente adolescentes y adulto en los que son más frecuentes la presencia de taquicardia paroxísticas supraventriculares (TPSV) manejadas con adenosina, arritmias ya sean éstas fibrilación auricular, síndrome WPW en el que está terminantemente prohibido el uso de digoxina, flutter e incluso taquicardia ventricular habitualmente, éstos últimos manejados con betabloqueantes (propanolol), amiodarona y flecainida, fármacos que resultan muy útiles en la práctica, todo esto en relación al trazado de base. El tratamiento quirúrgico por otro lado dependerá de la edad, forma anatómica y por tanto clínica del paciente. En recién nacidos sintomáticos con clínica de bajo gasto, requerirá de una cirugía precoz de reparación ya sea ésta univentricular o biventricular, la intervención precoz en neonatos graves con ventrículo derecho hipoplásico se hará mediante la operación de Starnes, luego se realizará el procedimiento de Glenn bidireccional, posteriormente la operación de Fontan, aunque en algunos de estos paciente se deberá considerar la opción de trasplante cardíaco. Mientras que en pacientes adolescentes y adultos sintomáticos con ICT igual o mayores de 0.65 es aconsejable la sustitución biológica o mecánica de la válvula tricúspide y en asintomáticos elegir un tratamiento conservador. A diferencia de los pacientes con episodios recidivantes de taquicardia supraventricular incesante y/o síndrome WPW, en los que es 
recomendable el estudio electrofisiológico y ablación de la vía accesoria. Se plantea la exéresis quirúrgica de la vía accesoria en el momento de la intervención en casos muy concretos de taquicardia inmanejable. El inconveniente es que aún luego de la reparación quirúrgica de Ebstein, la arritmias prevalecen en 10-20\% de los pacientes, por lo que se recomienda la limitación de actividades físicas de esfuerzo, además no existe evidencia de que la cirugía disminuya el riesgo de muerte súbita, por eso se reserva solo para pacientes sintomáticos. $(\mathbf{7 , 8 , 9 , 1 0 )}$

\section{Comentarios.}

La anomalía de Ebstein categorizada dentro de las cardiopatías congénitas raras de baja incidencia, es definida como el desplazamiento apical en el ventrículo derecho de la inserción de los velos posterior y septal de la válvula tricúspide, provocando grados variables de displasia y cambios en la clínica del paciente, también relacionada a la existencia o no de otras malformaciones acompañantes. Partiendo de aquí es importante recalcar que la mortalidad de los pacientes con cardiopatías congénitas no intervenidas como es la Anomalía de Ebstein varía desde la alta incidencia de muerte intrauterina hasta pacientes adultos asintomáticos o con sintomatología leve, sin embargo también aprendemos sobre la rápida progresión de la enfermedad una vez iniciada, por lo que se proponen estrategias de manejo del paciente sintomático y las diferentes pautas que debemos tener claras a la hora de tratarlo siempre en base de su clínica ,lo que nos presenten los exámenes complementarios sobretodo los trazados del electrocardiograma para un tratamiento inmediato, y la respuesta que presente el paciente a los tratamientos propuestos. A pesar de esto la evidencia nos enseña que incluso con la intervención quirúrgica no podemos asegurar al paciente que disminuya el riesgo de muerte súbita, lo que puede ocasionar negativa al tratamiento; como pudimos evidenciar en el relato de nuestro caso clínico.

\section{Conclusiones.}

- Concluimos que a pesar de la baja incidencia de la Anomalía de Ebstein en neonatos, y mucho más en adultos, es posible reconocerla, mediante una buena historia clínica que nos ayudará a eliminar muchos diagnósticos diferenciales, y la relación de los 
hallazgos al examen físico con los de los exámenes complementarios básicos que se realizan en cualquier emergencia como son: electrocardiograma y radiografía de tórax, para que con la sospecha clínica podamos confirmar la patología mediante el ecotranstorácico. Recordar la rápida progresión de la enfermedad sin importar la edad de inicio, para que nuestras propuestas de tratamiento vayan acorde al riesgo de mortalidad del paciente, y sobretodo entender que incluso con la propuesta adecuada, el paciente mantiene el derecho de decidir sobre su cuerpo, aún cuando sabemos el desenlace.

\section{Referencias bibliográficas.}

1. Jordán-Ríos, A., Magana Bailon, E., Martinez Aguilar, M, (2014). Anomalía de Ebstein Trabajo de revisión. Revista Mexicana de Cardiología. México.

2. Jordán-Ríos, A., Magana Bailon, E., Martinez Aguilar, M. (2014). Ebstein's anomaly. Revista Mexicana de Cardiología. México.

3. Begoña, B., Josephson, M. E. c (2012). Taquicardia ventricular en la enfermedad coronaria. Revista Española de cardiología. España.

4. Garrido Martín, A., Garrido Martín, J., Domínguez Melcón, F., González, A. E., Sobrino Daza, J. (2000) Displasia congénita de la válvula tricúspide (Ebstein like) en un varón de 73 años con insuficiencia tricuspídea severa. Revista Española de Cardiología. España.

5. A.J. Armengol Rofes, M. Serrano Durán, D.C. Albert Brotons, C. Sánchez López, J. Casaldáliga Ferrer, J.M. Girona Comas. (1996). Anomalía de Ebstein de la válvula tricúspide. A propósito de 35 casos. Anales Españoles de pediatría. Vol. 44 № 2 . . España.

6. Cove Point Foundation.Helen B. Taussig Children's Heart Center. Johns Hopkins University. (2017) Congenital Heart Disease. Spanish Translations- Faerron, J., Mas, C. Scientific Software Solutions. E.E.U.U.

7. Parisi Yanet, C. N. (2015) Caso clínico: Anomalía de Ebstein en el adulto ¿Qué podemos hacer?.Sociedad de Cardiología de Corrientes. España.

8. Olive Ruiz, J. M., Mateos García, M., Bret Zurita, M. (2003). Evaluación de cardiopatías congénitas en el adulto. Revista Española de Cardiología. España. 
9. Guerra, F. E., López, A., Escobar, V., (2010), Anomalía de Ebstein “tipo B” Caso clínico. Avances Cardiología. Vol. 30 No 4. España.

10. Martínez García, G., Montero García, Raciel, Cruz Rodríguez, Liz., Cárdenas Fernández, Y. Hernández González, A., (2009) Anomalía de Ebstein en edad adulta. Revista Cubana Médica. Cuba.

11. Bartrons Casas, J., (2015) Anomalía de Ebstein. Capítulo 30. DESCRIPCIÓN DE LA CARDIOPATÍA.https://docplayer.es/8214978-Anomalia-de-ebstein-capitulo-30joaquin-bartrons-casas-descripcion-de-la-cardiopatia.html 
Para citar el artículo indexado.

Montesdeoca S., Vera G., Aguirre F., Valdiviezo E. \& Zambrano A. (2018). Displasia congénita de la válvula tricúspide (anomalía de ebstein). A propósito de un caso en varón de 40 años. Revista electrónica Ciencia Digital 2(4), 219-230. Recuperado desde: http://cienciadigital.org/revistacienciadigital2/index.php/CienciaDigital/article/view/221/19 $\underline{4}$

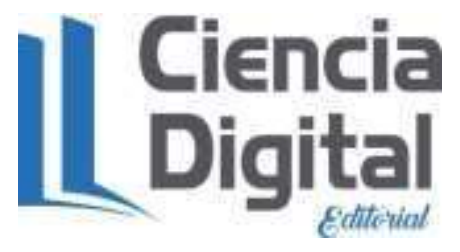

El artículo que se publica es de exclusiva responsabilidad de los autores y no necesariamente reflejan el pensamiento de la Revista Ciencia Digital.

El articulo queda en propiedad de la revista y, por tanto, su publicación parcial y/o total en otro medio tiene que ser autorizado por el director de la Revista Ciencia Digital.
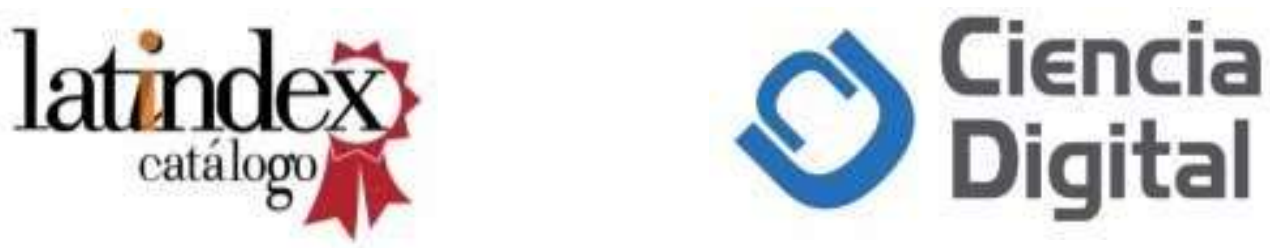